\title{
TECHNIQUE
}

\section{Displaying the long-term progression of patients with coronary artery disease}

\author{
C Sherlaw-Johnson, J Mitchard, S Gallivan, D L H Patterson, T Treasure
}

\begin{abstract}
Cardiac clinicians are often faced with the problem of trying to assimilate details of a patient's long-term history. Case notes are often lengthy, making this process difficult if not impossible in the time available during an outpatient examination. A computer system has been developed to assist with this task. This generates a graphical summary of the principal features of a patient's longterm progression. It gives an overview of how the patient's anginal status has changed, his or her drug treatment and any surgical interventions. The system also allows the clinican to display summaries of diagnostic tests carried out.

The system can be used to assist clinical management and speed up outpatient examination. It can also be used to facilitate case conference sessions and has potential for being used in medical education.
\end{abstract}

\section{(Br Heart f 1995;74:559-562)}

Keywords: coronary artery disease, data recording procedures, computer graphics outlining cardiac history.

Since 1989 we have been conducting a research programme aimed at investigating the long-term progression of patients with coronary artery disease.

As part of this research, case notes for all patients currently attending outpatient clinics in the cardiac department of the Whittington Hospital have been scrutinised. The task of retrospectively extracting information from such notes was found to be very laborious and often key facts, such as assessment of patients' anginal status or their medication, were either not recorded or recorded in a non-standard fashion making analysis difficult.

Typically, a patient with coronary artery disease attends outpatients clinics for many years. In addition, it is common that such patients may have had other referrals to the hospital for the treatment of other conditions. Consequently, the notes for an individual patient usually comprise a weighty folder containing among other things, hand written histories, laboratory test results, discharge summaries, general practitioners' letters, electrocardiogram (ECG) exercise test information, and summaries of angiograms. There is also associated correspondence between physicians and surgeons in cases involving coronary artery bypass grafting.

In view of the large quantity of information contained in a patient's notes it is often difficult to discover how a patient has progressed during the course of his or her disease. For example, trying to find the results of a patient's exercise ECG tests to see whether exercise tolerance has deteriorated can result in time-consuming sorting through the notes. Differences between the ways in which clinicians record information can also make it difficult to interpret what has been happening to a patient. So, for example, trying to find how long a patient has been treated with $\beta$ blockers may be hampered if, at some outpatient examinations, the information has not been recorded.

The fact that basic information about a patient's long-term progression can be hard to extract from a set of notes can lead to ineffective use of clinicians' time and a reduction in the quality of clinical management. In addition, as extracting systematic data from notes about a single patient is in itself a difficult task trying to summarise the progression of groups of patients, for audit purposes, say, becomes impractical.

This paper describes data recording procedures that have been established at the cardiac department of the Whittington Hospital. They are simple to operate and do not add substantially to clinicians' workload.

A computer system has been developed to assist with the analysis of the gathered data. Among other things, this allows a graphical summary to be produced outlining the principal features of an individual patient's longterm progression. This gives clinicians an immediate overview of a patient's cardiac history that is otherwise impractical to obtain.

\section{Data gathering}

Since June 1989 data concerning patients with coronary artery disease have been gathered prospectively at the Whittington Hospital. Information is recorded for every visit made to the outpatients clinic, every cardiac investigation and any bypass procedure 
Figure 1 Data collection form used during routine outpatient examinations.

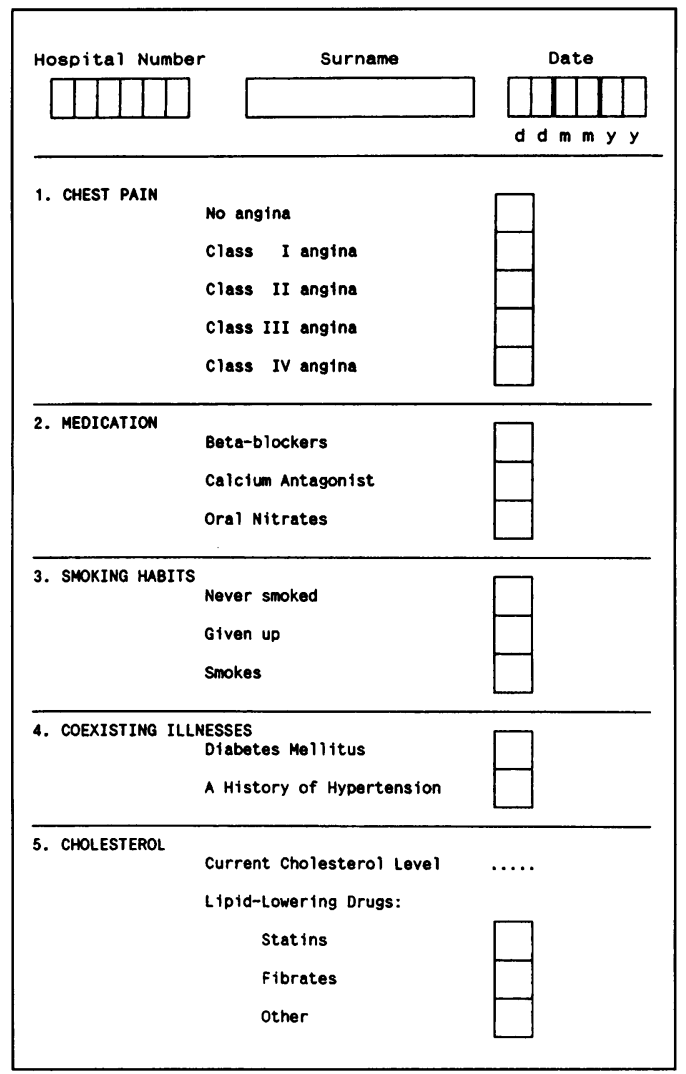

carried out. Two of the authors (JM and CSJ) have also retrospectively collected corresponding data from past case notes for all patients currently attending cardiac clinics.

Efforts have been made to keep the amount of information recorded as small as possible to avoid increasing the workload of the clinicians. The information noted at each session concerns assessments which would, in any case, form part of a routine cardiac examination. The only additional effort required by the clinicians is that they complete a short checklist during examinations concerning the status of the patient. For example, in the case of outpatient examinations, the clinician is
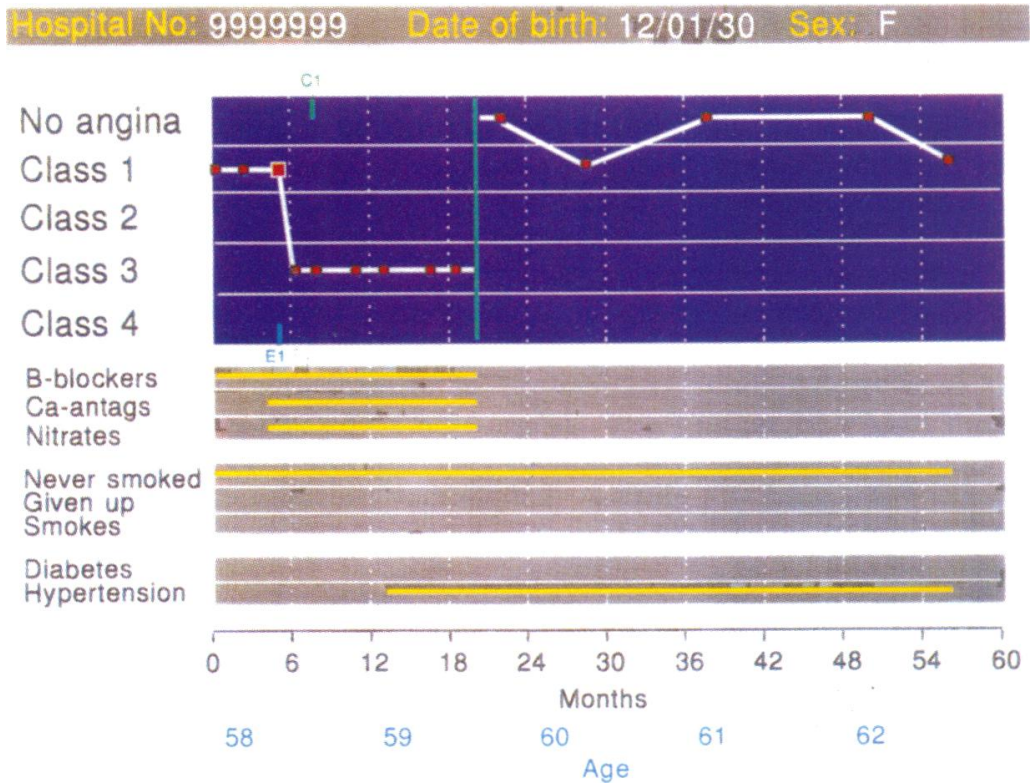

Figure 2 Typical graphical summary of the clinical data recorded for one patient. required to record at most 11 items of data.

Figure 1 shows the patient status form completed during an outpatient examination. Before attendance at the clinic such forms are attached to the patient's notes by clerical staff who fill in details of the patient's name, hospital number, and the date of the examination. During examination the clinician assesses the patient's class of angina, according to the Canadian Cardiovascular Society classification for angina pectoris (Appendix). The patient's current drug treatment is indicated by ticking appropriate boxes on the form, although specific information concerning dose levels and so forth is not included. Patients' smoking habits, their cholesterol level and whether they have a history of hypertension or diabetes are also indicated.

Similar data are collected for other investigations such as ECG exercise tests and coronary angiograms. For patients in whom coronary artery bypass grafting has taken place a brief summary of the principal features of the surgical procedure is also recorded.

Information from the forms is entered onto the department's clinical database system. This has been carried out by one of the researchers from the team, although this process is soon to be integrated with other clerical tasks carried out within the department.

\section{Displaying the progression of an individual patient}

Though little information is recorded about each patient contact, assimilating the whole of a patient's history is difficult because of the numerous examinations that are likely to have occurred. To assist this, a computer system has been developed that enables clinicians to examine the progression of a patient since they first attended the hospital. The computer system operates on an IBM compatible microcomputer, making use of colour graphics facilities.

The computer system allows the clinician to display patient information at various levels of detail. The system has been designed to be simple to operate and makes use of menu displays from which the user can choose particular options using cursor controls.

When the system is first entered, the user is presented with a preliminary menu of options. The usual starting option is to select a patient. This is achieved by either typing in the hospital number or selecting from a displayed list of hospital numbers available. The system displays a graphical summary of the selected patient's history.

Figure 2 shows a typical display. The top line of information lists the patient identification number, date of birth, and sex. The remainder of the display summarises the principal features of the patient's progression as time charts. Two time axes appear on the bottom of the display, one giving the time since the patient's first contact with the cardiac department, the other giving the 


\section{Losola No: 9999999 Date of bint $12101 / 30$. Sex: F}

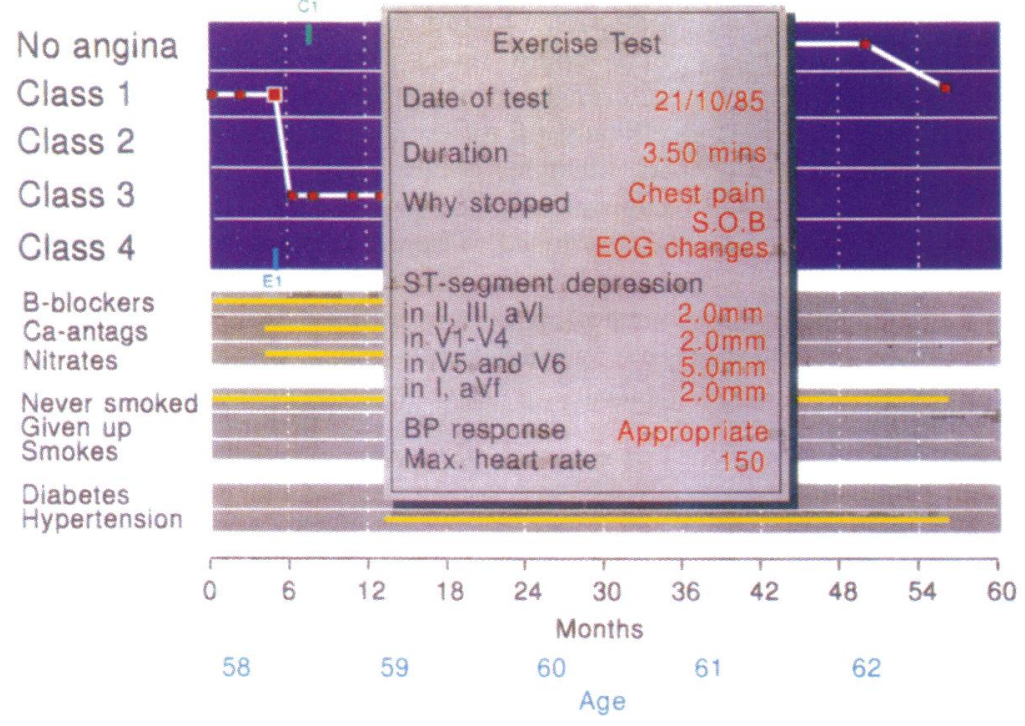

Figure 3 Display of exercise electrocardiogram test information.

patient's age in years. Above this, with a grey background, the presence of coexisting hypertension or diabetes and the patient's smoking history are displayed. Above these, an indication is given of the classes of drug treatment prescribed and the time periods involved. The final part of the screen displays the patient's progression. This is indicated by a trace, shown in white, showing the patient's anginal status at successive outpatient examinations, indicated as dots along the trace. Several symbols are used to indicate other events or procedures. For example, a red square indicates that the patient suffered myocardial infarction and a vertical green line indicates that coronary artery bypass has been performed. The number of times that angiographic catheterisation or ECG exercise testing was carried out is indicated by mnemonic codes.
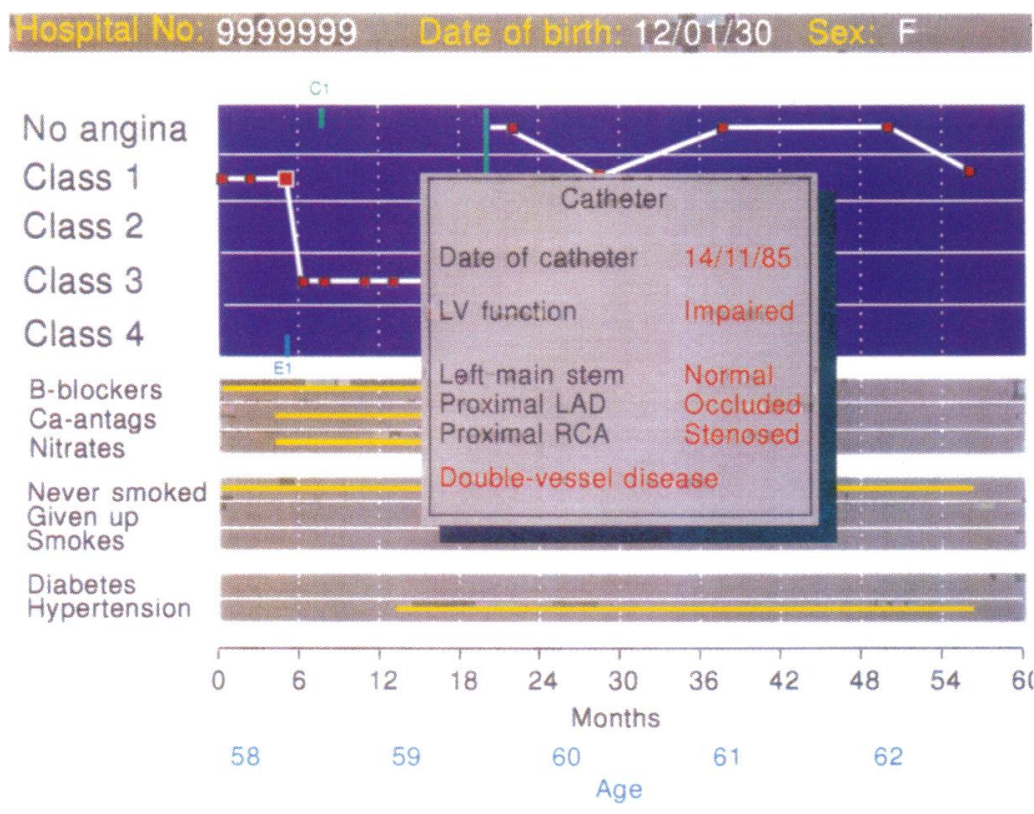

Figure 4 Display of cardiac angiogram findings.
As can be seen from the example display, this presents a concise summary of a patient's progression. In the case shown, the patient had an infarction six months after first contact with the cardiac department. Subsequently, her fairly severe angina failed to respond to drug treatment. Exercise ECG and angiographic tests indicated suitability for coronary artery bypass surgery. This was successful in relieving the patient's symptoms and drug treatment was no longer necessary. The patient continues to be followed up.

Considerable detail has been sacrificed to present information in this way so facilities have been added enabling the clinician to examine the patient's clinical history in more detail. Pressing the 'escape' key of the keyboard, causes a menu to be displayed listing items for which further information can be examined, including summaries of any cardiac angiograms, ECG exercise tests, cholesterol management, or surgical procedures. In each case, the information available takes the form of a brief summary. An example shown in fig 3 gives details of the findings from the patient's exercise ECG test, which in this case indicates ominous ST segment depression. If a patient has had several exercise ECG tests, details of each can be displayed in turn. In a similar fashion, details of findings from cardiac angiograms can be displayed. If a coronary bypass procedure has been performed then principal features of the surgical procedure are available: date of the procedure, the types of grafts, and the territories grafted (fig 5).

\section{Discussion}

The data collection system that has been developed enables long-term information concerning patients with coronary artery disease to be recorded in a systematic fashion. The methods used are simple to implement and do not add unduly to the tasks of busy clinicians. The recorded data comprises information that is readily available as part of the routine examination process. The only new procedure that has to be adopted is that clinicians fill in a checklist of their assessments for each patient contact. Recording information in such a systematic fashion ensures that a uniform record is available of the principal features of patient's cardiac status for every visit.

A major advantage of recording information in such a systematic fashion is that clinicians can examine a patients' long-term progression. The developed computer system assists in this objective. The graphical summaries give an immediate overview of a patient's history without the necessity of having to read through and assimilate voluminous case notes.

There is considerable potential for using such summary information. Ready access to a summary of a patient's history can clearly assist clinical management. Such summaries can also have a useful role in case conference sessions, giving a succinct precis of a patient's past condition. 


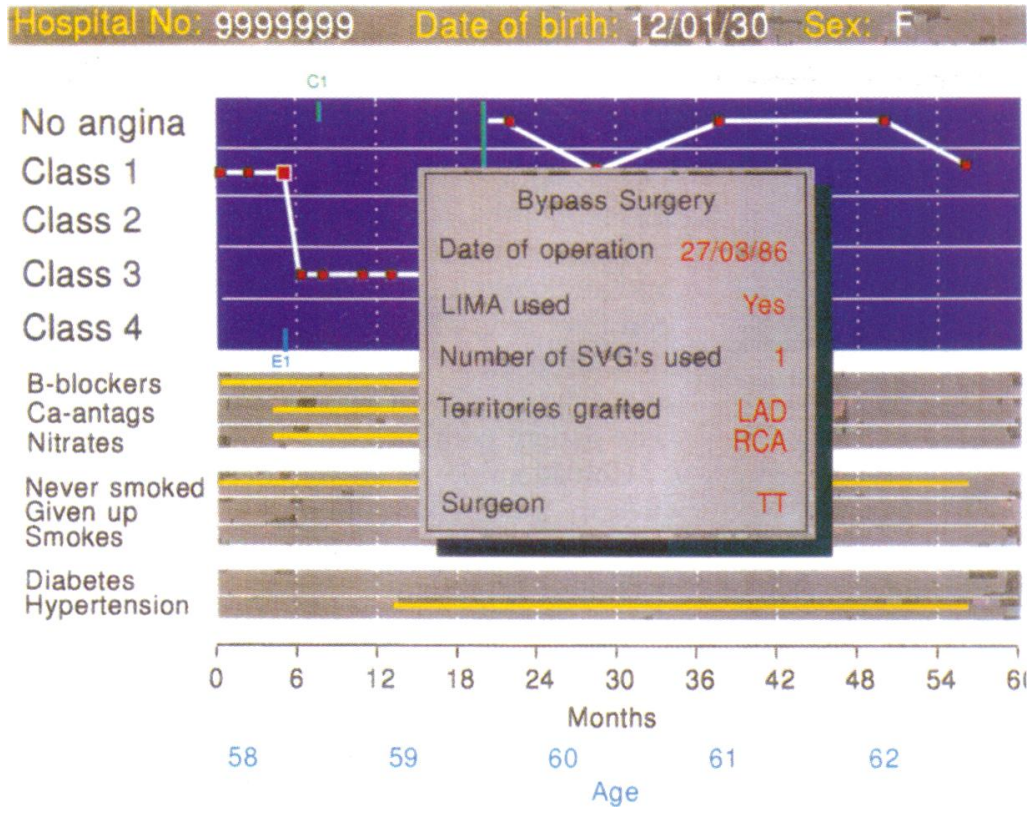

Figure 5 Summary of a coronary artery bypass procedure.

An important role is envisaged for using the results of this work in medical education. A large database of cases has now been compiled. This can be used to demonstrate to students the large patient-to-patient variation that exists in the long-term progression of coronary artery disease. By using the computer system medical students can browse through patient summaries and gain experience of typical cases seen in a specialist cardiac centre.
There is also the possibility that such summaries could be of more direct benefit to patients. During their examination the clinician could review their case making direct reference to their displayed summary. The efficacy of different treatments and changes in lifestyle could then be discussed with examples showing other cases suitably anonymised. There are plans to give patients attending the Whittington Hospital a printed version of their summary charts. The benefits that would result from this remain to be tested.

\footnotetext{
Appendix

Canadian Cardiovascular Society classification for angina pectoris:

Class I-Ordinary physical activity such as walking or climbing stairs does not cause angina. Angina with rapid or prolonged exertion at work or recreation.

Class II-Slight limitation of ordinary activity. Walking or climbing stairs rapidly, walking uphill, walking or stair climbing after meals, or in cold, or in wind, or under emotional stress, or only after the few hours after awakening. Walking more than two blocks on the level and climbing more than one flight of stairs at normal pace and in normal conditions.

Class III-Marked limitation of ordinary physical activity. Walking more than two blocks on the level and climbing more than one flight of stairs at normal pace and in normal conditions.

Class IV-Inability to carry on any physical activity without discomfort-anginal syndrome may be present at rest.
} 